\title{
Thermal effect in laser perforation of liver
}

\author{
Olga I. Baum*, Alexander K. Dmitriev, Vladimir N. Kortunov, Olga A. Tiflova, \\ and Valery A. Ulyanov \\ Institute of Photonic Technologies, Federal Scientific Research Centre 'Crystallography and Photonics' of the Russian \\ Academy of Sciences, 2 Pionerskaya Str., Moscow (Troitsk) 142190, Russia \\ * e-mail: baumolga@gmail.com
}

\begin{abstract}
One of the trends in the development of modern regeneration medicine is the application of high-intensity laser radiation for deep perforation of biological tissues, stimulating the regeneration of structure and functions of the pathologically changed tissues. In the paper, the possibility to control the location and parameters of the biotissue thermal stimulation zones is studied theoretically and experimentally, depending on the conditions of laser perforation in application to the liver laser-induced regeneration problem. The results of numerical simulation revealed the basic trends in the behaviour of the thermal stimulation zone, which are in good agreement with the experimental data on laser perforation of soft biotissues ex vivo using an optical fibre. (C) 2018 Journal of Biomedical Photonics \& Engineering.
\end{abstract}

Keywords: fibre laser; perforation; liver; regeneration; thermal fields.

Paper \#3288 received 11 Apr 2018; revised manuscript received 25 May 2018; accepted for publication 25 May 2018; published online 17 Jun 2018. doi: 10.18287/JBPE18.04.020302.

\section{References}

1. I. I. Berishvili, Transmyocardial Laser Revascularisation, GEOS, Moscow, Russia (2016) [in Russian]. ISBN 978-5-89118-703-0

2. X. M. Mueller, H. T. Tevaearai, C. Y. Genton, P. Chaubert, and L. K von Segesser, "Are there vascular density gradients along myocardial laser channels?" The Annals of Thoracic Surgery 68(1), 125-129 (1999).

3. X. M. Mueller, H. T. Tevaearai, C. Y. Genton, P. Chaubert, and L. K von Segesser, "Improved neoangiogenesis in transmyocardial laser revascularization combined with angiogenic adjunct in a pig model," Clinical Science 99(6), 535-540 (2000).

4. D. I. Alyokhin, A. A. Fokin, "Prospects of using high-intensity laser radiation for the treatment of chronical ischemia of extremities," Patologiya krovoobrashcheniya i kardiokhirurgiya 2, 88-92 (2005) [in Russian].

5. S. V. Kapralov, I. A. Mel'nikova, U. G. Shapkin, and V. V. Alipov, "Experimental modelling of laser fenestration of liver," Byulleten' meditsinskikh Internet-konferentsii 1(2), 47 (2011) [in Russian].

6. I. A. Shved, T. E. Vladimirskaya, A. V. Vorobey, O. P. Shorez, S. V. Alexandrov, and A. C. Shuleiko, "Liver tissue regeneration after laser coagulation," Zdravookhraneniye (Belarus) 3, 4-8 (2014) [in Russian].

7. L. Lamalice, F. Le Boeuf, and J. Huot, "Endothelial cell migration during angiogenesis," Circulation Research 100(6), 782-794 (2007).

8. A. Briolay, R. Jaafar, G. Nemoz, and L. Bessueille, "Myogenic differentiation and lipid-raft composition L6 skeletal muscle cells are modulated by PUFAs," Biochimica et Biophysica Acta (BBA) - Biomembranes 1828(2), 602-613 (2013).

9. A. Dolganiuc, "Role of lipid rafts in liver health and disease," Would Journal of Gastroenterology 17(20), 2520-2535 (2011).

10. M. Troyanova-Wood, J. D. Musick, B. L. Ibey, R. J. Thomas, and H. T. Beier, "Observation of changes in membrane fluidity after infrared laser stimulation using a polarity-sensitive fluorescent probe," Proceeding of SPIE 8941, 89410I (2014).

11. L. Jin, C. Millard, J. P. Wuskell, H. A. Clark, and L. M. Loew, "Cholesterol-enriched lipid domains can be visualized by di-4-ANEPPDHQ with linear and nonlinear optics," Biophysical journal: biophysical letters 89(1), L04-L06 (2005). 
12. A. J. Garcia-Saez, S. Chiantia, and P. Schwille, "Effect of line tension on the lateral organization of lipid membranes," Journal of Biological Chemistry 282(46), 33537-33544 (2007).

13. G. Muller, K. Dorschel, and H. Kar, "Biophysics of photoablation process," Lasers in Medical Science 6(3), 241-254 (1991).

14. N. N. Kalitkin, Numerical Methods, Nauka, Moscow, Russia (1978) [in Russian].

15. O. I. Baum, “Temperature control system for laser reshaping of nasal septum," Izvestiya Vysshikh Uchebnykh Zavedeniy. Priborostroenie 58(10), 847-854 (2015) [in Russian].

16. O. I. Baum, Y. M. Soshnikova, E. N. Sobol, A. Ya. Korneychuk, M. V. Obrezkova, V. M. Svistushkin, O. K. Timofeeva, and V. V. Lunin, "Laser reshaping of costal cartilage for transplantation," Lasers in surgery and medicine 43(6), 511-515 (2011).

17. A. V. Yuzhakov, A. P. Sviridov, E. M. Shcherbakov, O. I. Baum, and E. N. Sobol, "Optical properties of costal cartilage and their variation in the process of non-destructive action of laser radiation with the wavelength $1.56 \mu \mathrm{m}$," Quantum Electronics 44(1), 65 (2014).

18. O. I. Baum, E. N. Sobol, A. V. Bolshunov, A. A. Fedorov, O. V. Khomchik, A. I. Omelchenko, and E. M. Shcherbakov, "Microstructural changes in sclera under thermo-mechanical effect of $1.56 \mu$ m laser radiation increasing transscleral humor outflow," Lasers in surgery and medicine 46(1), 46-53 (2014).

19. O. Baum, S. Wachsmann-Hogiu, T. Milner, and E. Sobol, "Laser-assisted formation of micropores and nanobubbles in sclera promote stable normalization of intraocular pressure," Laser Physics Letters 14(6), 065601 (2017).

20. E. Sobol, A. Shekhter, A. Baskov, O. Baum, I. Borchshenko, V. Golubev, A. Guller, I. Kolyshev, A. Omeltchenko, A. Sviridov, and O. Zakharkina, "Regeneration of spine disc and joint cartilages under temporal and space modulated laser radiation," Proceeding of SPIE 7179, 71790B (2009).

21. E. Sobol, A. Shekhter, A. Guller, O. Baum, and A. Baskov, "Laser-induced regeneration of cartilage," Journal of Biomedical Optics 16(8), 080902 (2011).

22. E. Sobol, O. Baum, A. Shekhter, S. Wachsmann-Hogiu, A. Shnirelman, Y. Alexandrovskaya, I. Sadovskyy, and V. Vinokur, "Laser-induced micropore formation and modification of cartilage structure in osteoarthritis healing," Journal of Biomedical Optics 22(9), 091515 (2017).

23. M. N. Libenson, E. B. Yakovlev, and G. D. Shandybina, "Interaction of Laser Radiation with Matter (Power Optics)," Lecture Notes. Part II. Laser Heating and Material Destruction, V. P. Veyko (Ed.), ITMO, SaintPetersburg (2006) [in Russian].

\section{Introduction}

One of the trends in the development of regeneration medicine is the application of high-intensity laser radiation for stimulating the recovery of tissue blood supply, structure and functions of vital organs of the human organism. Such approach, in particular, allowed the development of a new method of treatment of the ischemic heart disease, the transmyocardial laser revascularization revascularisation, in the process of which deep channels are created in the ischemic muscle by the high-power laser action. The laser perforation of the cardiac muscle initiates the process of angiogenesis, i.e., the growth of microcapillaries in the zone of the laser impact. The mechanisms of laser revascularisation include the following: i) the development of aseptic inflammation in the perforated region in response to the laser impact with subsequent regeneration and development of microcapillaries; ii) the thermal impact on the tissues surrounding the laser channel, activating different cell elements that are sources of growth factors playing the key role in simulating the angiogenesis process; iii) the formation of multiple microscopic ruptures of the tissue in the channel walls caused by the shockwave impact that establishes a new level of oxygen consumption in the affected volume, improving the blood supply. To date it is established that the improvement and long-term stability of the patient's clinical indicators depend both on the radiation wavelength and on the mode of laser tissue perforation (continuous-wave, pulsed, etc.), which determine the positive effect of the procedure [1]. It is experimentally shown that the neovascularisation of the ischemic myocardium is observed both directly in the region of the laser channel [2], and within 3-5 $\mathrm{mm}$ zone around the channel [3], thus essentially increasing the revascularisation domain in the ischemic myocardium.

The method of laser perforation is also developed in application to other human tissues and organs. The positive effect of laser revascularisation is known for the ischemic muscles of lower extremities, where it is implemented via an optical fibre with the lasers at the wavelength 0.98 and $1.06 \mu \mathrm{m}$ [4]. After the laser optical fibre perforation of cirrhotic rat liver (wavelength $1.06 \mu \mathrm{m}$ ), the regeneration of the hepatic tissue with the signs of new microcapillaries and gall ducts formation in the region of laser channels was observed $[5,6]$. These studies were carried out according to the following scheme: the laser perforation of the biological object was carried out with the specified radiation power and exposure time. Then the histochemical and microscopic study of the tissue sections was performed, and the relation between the laser radiation parameters (power, exposure time) and the structural changes of the tissue was established. However, no information on the 
laser-induced effects in the perforated region for these tissues is available. Other types of lasers are not used for these purposes at present time.

The thermal stimulation of angiogenesis is considered as the basic model of myocardium revascularisation using laser perforation [1]. The target for the laser-induced thermal angiogenesis induction can be the cell membranes. The interaction with the membrane short-lived microscopic domains (rafts) is a necessary stage of activating the key enzymes controlling the angiogenesis [7]. The differentiation of myoblasts in the process of muscle fibre regeneration is probably caused by the possibility of raft structure formation in the cell membranes [8]. The functioning and interaction of different types of cells comprising the liver in the process of regeneration is also largely dependent on the possibility of thermal induction of rafts in them [9]. The IR laser radiation enhances the fluidity of lipids in cell membranes in vitro [10], facilitating the modification of their microdomain raft structure [11]. The temperature of the phase transition of cell membranes from the unordered state to the ordered one depends on their composition and for natural lipids ranges from 38 to $66^{\circ} \mathrm{C}$ [12]. The creation of temperature fields initiating thermal induction of raft transition around the laser channels is a promising approach to the development of laser stimulation method for the regeneration of liver and muscle tissue.

The present paper is aimed at the study of the possibility to determine and control the location and parameters of the thermal stimulation zone in the tissue caused by laser perforation with an optical fibre at the wavelength $1.56 \mu \mathrm{m}$ in application to the problem of laser-induced liver regeneration.

\section{Materials and methods}

As a source of radiation, we used the fibre LSP laser with the wavelength $1.56 \mu \mathrm{m}$ (NPO "IRE-Polus") with the power up to $10 \mathrm{~W}$, operating in the continuous-wave mode. The power of radiation at the output face of the fibre $600 \mu \mathrm{m}$ in diameter was from 2 to $8 \mathrm{~W}$, the velocity of the fibre movement into the tissue was changed stepwise from $0.1 \mathrm{~mm} / \mathrm{s}$ to $1 \mathrm{~mm} / \mathrm{s}$. To measure the power at the fibre output we used the power meter (Coherent, Inc., USA).

The material for ex-vivo studies were the samples of liver from a one-year-old white pig.

The samples were placed on an immobile platform. The sample perforation was implemented by moving the fibre into the tissue using the motorised linear translator with the specified velocity. The straight-through perforation of biotissue samples was performed. After the perforation the samples were frozen, the longitudinal and transverse sections were prepared in the channel region, and the thermally damaged zone (TDZ) of the tissue was investigated microscopically around the laser channel, including the carbonisation and the coagulation zone (Fig. 1).

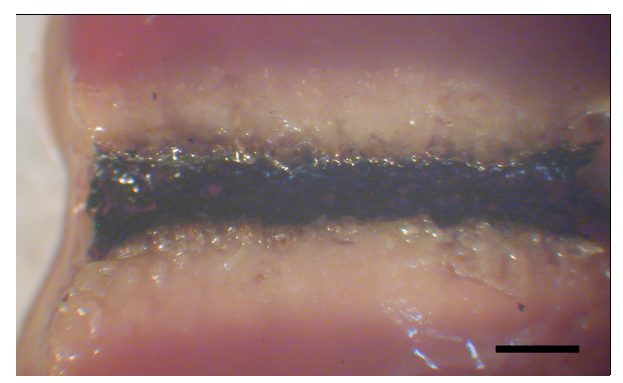

Fig. 1 A typical view of the thermally damaged zone (TDZ) of the tissue around the laser channel in the sample of liver in vitro after perforation with the optical fibre (the entrance of the fibre into sample occurs from the left), the scale bar corresponds to $1 \mathrm{~mm}$.

The TDZ width was measured as the distance from the laser channel wall to the boundary of the coagulation zone, visually observed as the whitish tissue, its colour being a result of coagulation under the heating in the process of perforation up to the temperature $70^{\circ} \mathrm{C}$ [13]. The microscopy of TDZs was implemented using the MBS-10 microscope with $100 \times$ magnification.

The width of the thermal stimulation zone (TSZ) in the process of tissue regeneration corresponded to the temperature range $38-66^{\circ} \mathrm{C}$ [12] and was determined using the calculation method according to the developed theoretical model.

The temperature distribution over the tissue surface was measured using an infrared imager, the infrared camera FLIR A655sc (FLIR Systems, Inc. Sweden), with the resolution of $640 \times 480$ pixels at $50 \mathrm{~Hz}$, $640 \times 120$ pixels at $200 \mathrm{~Hz}$, and the response time $8 \mu \mathrm{s}$, operating in the wavelength range from $7.5 \mu \mathrm{m}$ to $14 \mu \mathrm{m}$. The temperature sensitivity amounted to $50 \mathrm{mK}$ within the temperature range $\left(-20^{\circ} \mathrm{C}-+150^{\circ} \mathrm{C}\right)$. For comparison with the theoretical calculation, we measured the temperature in the process of fibre insertion into the sample at the outer side surface and at the face of the sample for the given distance from the fibre axis.

Fig. 2 presents a schematic diagram of the considered space domain, superimposed on the thermal image of the temperature distribution at the side surface of the biotissue sample separated from the fibre axis by the distance of $2-3 \mathrm{~mm}$. The temperature distribution was measured along and across the axis of the optical fibre motion. The transverse distributions were measured at the fixed distances of $2 \mathrm{~mm}$ (Section 1) and $8 \mathrm{~mm}$ (Section 2) from the entrance (left-hand) surface of the sample at the moments, when the optical fibre reached these marks. Correspondingly, for different velocities of the optical fibre movement into the tissue sample, the transverse distributions were measured at different time intervals from the beginning of irradiation. For example, for the velocity $0.2 \mathrm{~mm} / \mathrm{s}$ the time moments were $t_{1}=10 \mathrm{~s}$ and $t_{2}=40 \mathrm{~s}$; for the velocity $0.5 \mathrm{~mm} / \mathrm{s}$ they were $t_{1}=4 \mathrm{~s}$ and $t_{2}=16 \mathrm{~s}$. 


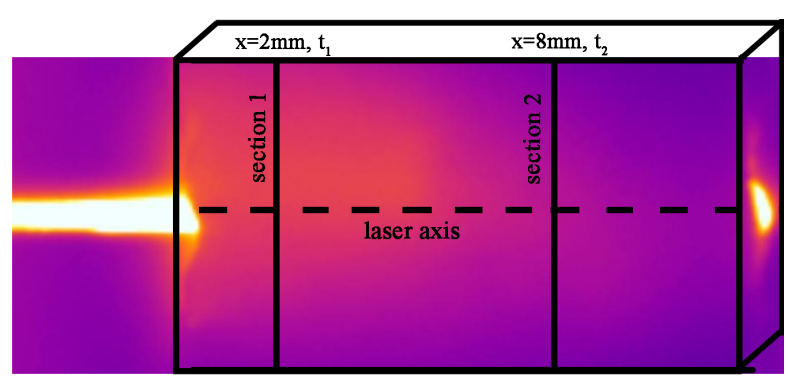

Fig. 2 Calculation scheme of moving the optical fibre into the tissue (from left to right) superimposed with the thermal image of temperature distribution. The boundaries of the biotissue sample and the sections 1 and 2 separated by 2 and $8 \mathrm{~mm}$ from the input surface of the sample are shown.

In the theoretical part of the study, in order to determine the thermal stimulation zones and their behaviour depending on the laser irradiation parameters, we constructed a theoretical model of heating the biotissue with the fibre, moving into it. The simulation of the liver tissue heating by the laser radiation is based on the heat conduction theory. We solved the heat conduction equation with the volume source, created by the laser radiation, damping with the depth according to the Booger-Lambert-Beer law, and expanding as the optical fibre is moved into the tissue.

The density of the incident energy flux at the biotissue surface, supplied by the optical fibre, has the Gaussian transverse spatial distribution with the effective beam radius that allows for the divergence of the beam with penetration depth. The divergence was approximated by a linear function determined from the comparison of numerical and experimental data in two sections, separated by the given distance.

For the numerical simulation, using the software package «Mathematica 5.0» the finite difference method and the Frankel-Dufort scheme were applied [14].

\section{Theoretical model of laser perforation of biotissues using an optical fibre}

The model is based on the heat conduction equation (1) with the moving source of heat $G(x, y, z, t)$, created by the laser radiation, delivered via the optical fibre with the diameter $600 \mu \mathrm{m}$, moved into the tissue with the velocity that can be varied from $0.1 \mathrm{~mm} / \mathrm{s}$ to $0.5 \mathrm{~mm} / \mathrm{s}$

$$
\frac{\partial T(x, y, z, t)}{\partial t}=a\left(\begin{array}{c}
\frac{\partial^{2} T(x, y, z, t)}{\partial x^{2}}+ \\
+\frac{\partial^{2} T(x, y, z, t)}{\partial y^{2}}+ \\
+\frac{\partial^{2} T(x, y, z, t)}{\partial z^{2}}
\end{array}\right)+G(x, y, z, t),
$$

where $a$ is the thermal diffusivity.

A similar heat source was considered and the detailed description of the influence of different parameters of laser radiation on the result of thermal heating was presented in the paper [15], devoted to the design of the control system aimed at the conservation of the nasal septum functional properties in the laser reshaping procedure. The action of a similar heat source on the tissues of tendons [16-17] and sclera [18-19] of the eye was studied with thermomechanical stresses taken into account, and in Refs. [20-22] with the analogous source at the wavelength $1.56 \mu \mathrm{m}$ the data on the biological tissue regeneration were obtained. The difference of the model used in the present paper from the earlier ones is the movement of the volume heat source.

In the present study, we solve the problem with moving source of radiation having the Gaussian distribution in the plane perpendicular to the direction of motion. The Booger-Lambert-Beer law with the effective absorption index $\kappa(2)$ describes the damping of radiation with the depth of propagation into the biological tissue. The density of the incident energy flux at the sample surface has the Gaussian spatial distribution with the effective beam radius $r_{0}(x)$. The radiation passed through the optical fibre generates a thermal field that propagates in the liver. Due to the inhomogeneity of the biological tissue and multiple scattering inside it, the Gaussian source of heat expands with the motion into the sample. This expansion is considered by introducing the dependence of the effective radius $r_{0}(x)$ upon the coordinate $x$. In the first approximation for numerical simulation, this dependence was taken linear

$G(x, y, z, t)=q(t, v) \exp \left[-\frac{y^{2}+z^{2}}{r_{0}(x)^{2}}\right] \frac{\kappa \exp (-\kappa x)}{c \rho}$

where $q(t, v)$ is the integral power of the laser radiation depending on time and the velocity of motion $v$ of the heat source, $c$ is the specific heat capacity, $\rho$ is the density.

The boundary condition (3) corresponds to the continuity of heat flux and temperature at the boundary between two media, $x=h_{m}$ :

$$
\begin{aligned}
& \lambda_{m} \frac{\partial T^{m}\left(h_{m}, y, z, \tau\right)}{\partial x}=\lambda_{m+1} \frac{\partial T^{m+1}\left(h_{m}, y, z, \tau\right)}{\partial x} \\
& T^{m}\left(h_{m}, y, z, \tau\right)=T^{m+1}\left(h_{m}, y, z, \tau\right)
\end{aligned}
$$

Here $\lambda_{m}$ is the heat conductivity, $m$ is the number of the considered layer.

For each considered layer of space $m$, the heat conduction equation can be written allowing for the form of the heat source and the reflection from the interface $K_{m}^{r}$ between the media 


$$
\begin{aligned}
& \frac{\partial T(x, y, z, \tau)}{d \tau}-a_{m}\left(\begin{array}{c}
\frac{\partial^{2} T(x, y, z, \tau)}{\partial x^{2}}+ \\
+\frac{\partial^{2} T(x, y, z, \tau)}{\partial y^{2}}+ \\
+\frac{\partial^{2} T(x, y, z, \tau)}{\partial z^{2}}
\end{array}\right)= \\
& =\left(1-K_{m}^{r}\right) q(t, v) \exp \left[-\frac{y^{2}+z^{2}}{r_{0}(x)^{2}}\right] \frac{\kappa \exp (-\kappa x)}{c_{m} \rho_{m}} .
\end{aligned}
$$

Here $c_{m}$ is the layer heat capacity, $\rho_{m}$ is its density, $\kappa$ is the light absorption index.

For the biotissue region where the boiling of water is not achieved, only the thermal source is taken into account in the right-hand side of Eq. (4). With the increase of exposure time and the achievement of boiling temperature, the heat expenditures for evaporation become essential. The bulk growth of temperature in this region decelerates and the specific flow carried away by the vapour approaches the mean value of $(0.7 \div 0.8) * q(t, v)$, according to Libenson [23], which allows a correction of the right-hand side of the heat conduction equation near $100^{\circ} \mathrm{C}$. In this case, a wave of evaporation propagates into the substance, the velocity of which increases, while the size of the heated domain $v t \approx \sqrt{a t}$ gradually decreases. When the wave of evaporation overtakes the thermal wave, the role of heat conductivity is reduced to the determination of temperature before the steady-state evaporation front. Thus, the problem of localising the heat simulation zone, where the processes of angiogenesis are triggered at the cellular level $\left(38-66^{\circ} \mathrm{C}\right)$ can be considered basing on Eqs. (1)-(3).

For the numerical modelling, we used the finite difference method and the three-layered Frankel-Dufort scheme for time variable. In the finite-difference form, the heat conduction equation for the numerical simulation is written as

$$
\begin{aligned}
& \frac{T_{i x, i y, i z}^{j+1}-T_{i x, i y, i z}^{j-1}}{2 d t}=
\end{aligned}
$$

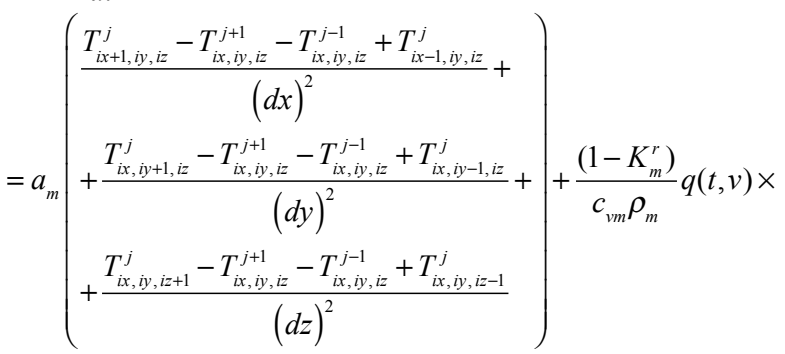

$$
\begin{aligned}
& \times \exp \left[-\frac{\left[(i y-1) d y-\frac{y_{\max }-y_{\min }}{2}\right]^{2}+\left[(i z-1) d z-\frac{z_{\text {max }}-z_{\text {min }}}{2}\right]^{2}}{r_{0}(x)^{2}}\right] \times \\
& \times \kappa_{m} \exp \left(-\kappa_{m}[i x-1] d x\right) \text {. }
\end{aligned}
$$

The temperature at the current step of the time evolution was expressed in terms of the temperature values at two preceding layers:

$$
T_{i x, i y, i z}^{j+1}=T_{i x, i y, i z}^{j-1} \frac{1-2 d t a_{m}\left(\frac{1}{(d x)^{2}}+\frac{1}{(d y)^{2}}+\frac{1}{(d z)^{2}}\right)}{1+2 d t a_{m}\left(\frac{1}{(d x)^{2}}+\frac{1}{(d y)^{2}}+\frac{1}{(d z)^{2}}\right)}+
$$

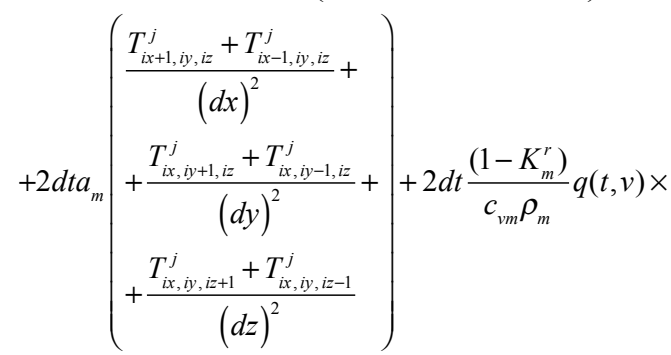$$
\times \exp \left[\frac{\left[(i y-1) d y-\frac{y_{\text {max }}-y_{\text {min }}}{2}\right]^{2}+\left[(i z-1) d z-\frac{z_{\text {max }}-z_{\text {min }}}{2}\right]^{2}}{r_{0}(x)^{2}}\right] \times
$$

$\times \kappa_{m} \exp \left(-\kappa_{m}[i x-1] d x\right)$.

The numerical problem was solved using the software package «Mathematica 5.0».

\section{Discussion and comparison of experimental and numerical simulation results}

Below we present the results of numerical simulation of temperature fields and the experiments with thermal imaging measurements of these fields at different velocities of the laser fibre moving into the tissue.

In the process of tissue perforation with the optical fibre, a layer of carbonised (charred) tissue is formed at the front face of the fibre, analogous to the carbonised zone around the channel. Its thickness at the fibre face was smaller than 80-100 $\mu \mathrm{m}$, while the size of carbonisation zone around the laser channel amounted to $20-100 \mu \mathrm{m}$ depending on the laser radiation power. As shown by direct measurements, the power change at the output fibre face covered with the carbonised tissue after the perforation does not exceed $7-10 \%$ of the initial power, i.e., not more than $10 \%$ of laser radiation is absorbed by the carbonised tissue at the fibre face. Therefore, in our calculations we did not take the absorption of radiation by the carbonised layer into account.

For the velocity of fibre motion $0.2 \mathrm{~mm} / \mathrm{s}$, we compared the experimental and theoretical temperature distribution curves (Fig. 3) and selected the rates of thermal source broadening with the depth, providing good agreement of theoretical and experimental results. The laser power at the face of the optical fibre was equal to $5 \mathrm{~W}$. 


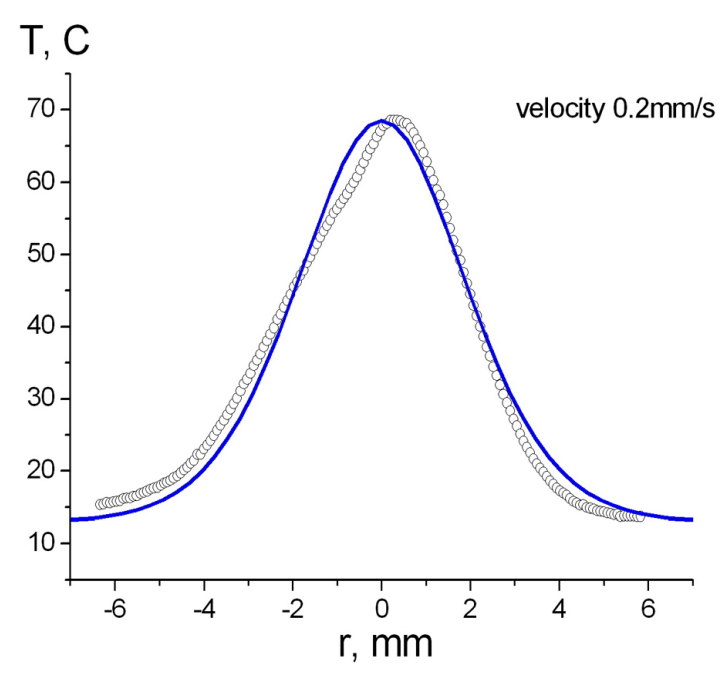

a

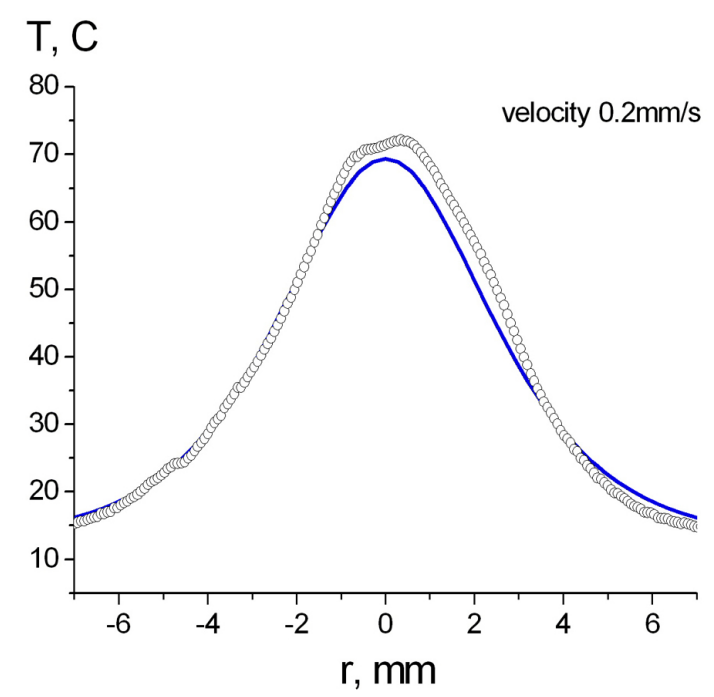

$\mathrm{b}$

Fig. 3 Temperature fields at the distance $2 \mathrm{~mm}$ (in $10 \mathrm{~s}$ after the beginning of perforation) (a) and $8 \mathrm{~mm}$ (in $40 \mathrm{~s}$ after the beginning of perforation) (б) from the sample surface for the velocity of the fibre $0.2 \mathrm{~mm} / \mathrm{s}$. The empty circles show the data of thermal imaging and the solid curves plot the simulation results.

Basing on the selected rate of the heat source expansion, the temperature distribution curves for the velocity $0.5 \mathrm{~mm} / \mathrm{s}$ were calculated and superimposed on the corresponding experimental curves. One can see good agreement between the computer simulation using the selected expansion rate of the heat source and the experiment (Fig. 4).

The temperature field was calculated at the back surface $20 \mathrm{~s}$ before the through perforation of the biotissue sample by the moving optical fibre. The results are confirmed well by the experimental data of thermal imaging (Fig. 5).

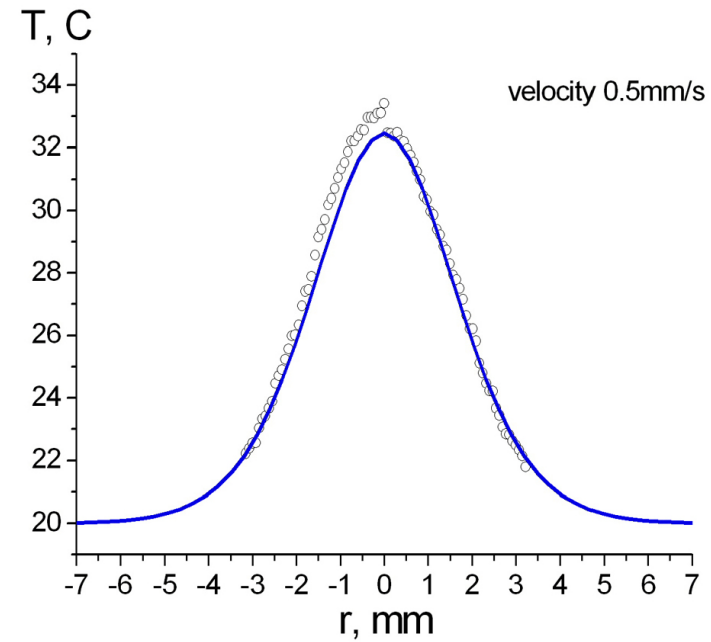

a

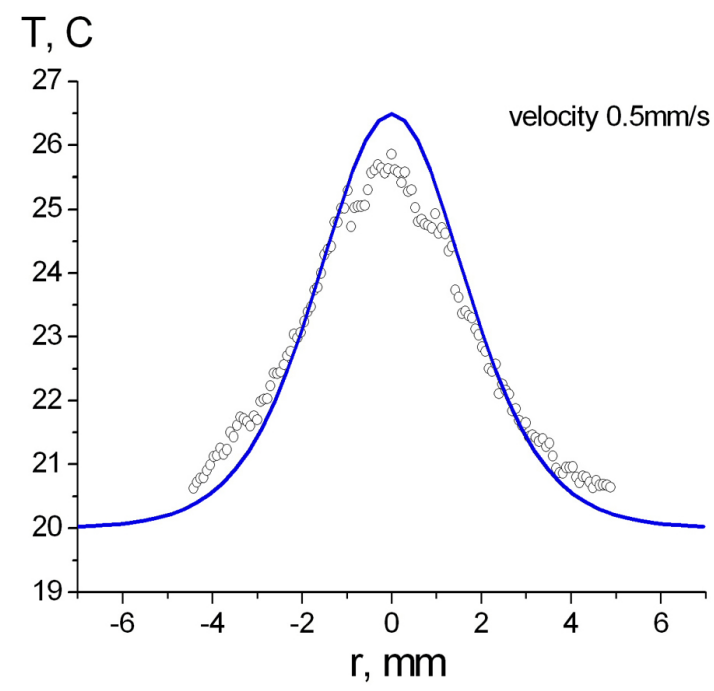

b

Fig. 4 Temperature fields at the distance $2 \mathrm{~mm}$ (in $4 \mathrm{~s}$ after the beginning of perforation) (a) and $8 \mathrm{~mm}$ (in $16 \mathrm{~s}$ after the beginning of perforation) (b) from the sample surface for the velocity of the fibre movement $0.5 \mathrm{~mm} / \mathrm{s}$. The empty circles show the thermal imaging data and the solid curves represent the simulation results.

We performed the calculation aimed at the determination of the TSZ position with respect to the axis of the laser channel and at the comparison with the experimental data. Fig. 6 presents the dependences of the TSZ width corresponding to the temperature range $38-66^{\circ} \mathrm{C}$ and the boundary of the zone, where the coagulation temperature was observed $\left(70^{\circ} \mathrm{C}\right)$ on the radiation power for the motion of the fibre into the tissue sample with the velocity $0.5 \mathrm{~mm} / \mathrm{s}$. For comparison, the experimental data for the boundary of coagulation zone are presented. 


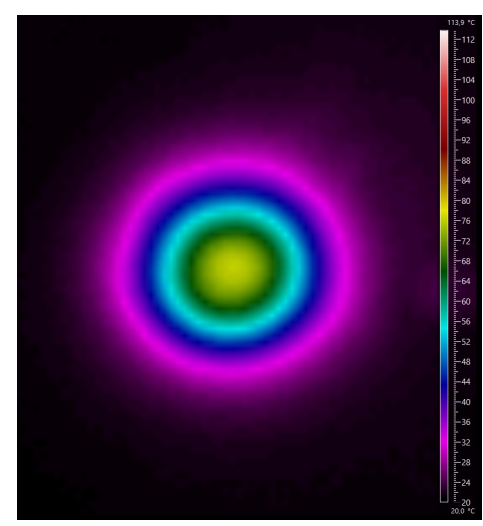

a

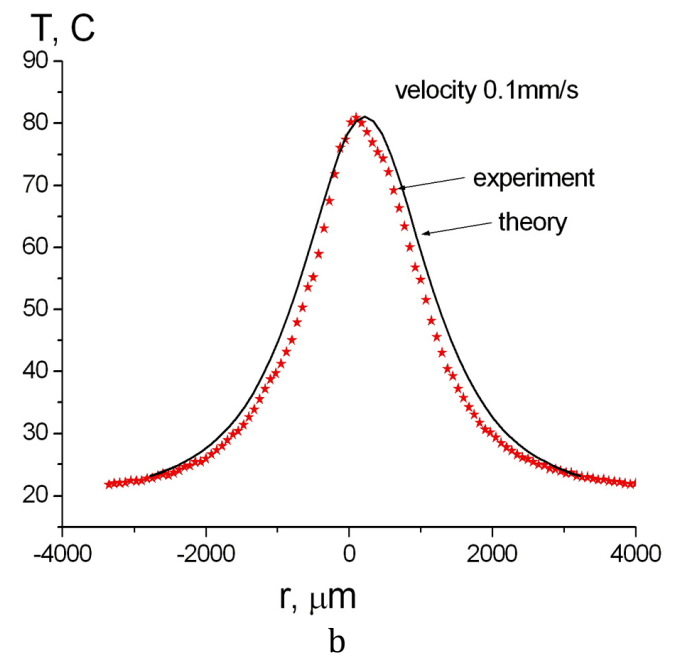

Fig. 5 (a) Temperature distribution at the back surface of the liver sample (perpendicular to the fibre axis) at the moment when the output face of the fibre moving with the velocity $0.1 \mathrm{~mm} / \mathrm{s}$ was at the distance of $2 \mathrm{~mm}$ from this surface. (b) The asterisks show the data of thermal imaging and the solid curve shows the results of simulation.

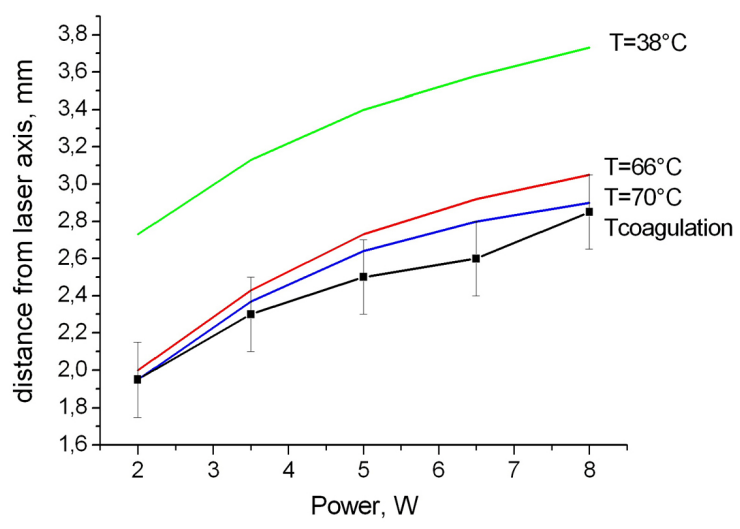

Fig. 6 Dependence of the TSZ width on the radiation power for the velocity of fibre movement into the biotissue sample equal to $0.5 \mathrm{~mm} / \mathrm{s}$. The experimental data for the coagulation zone boundary (Tcoagulation) are also presented.

Fig. 7 presents the calculated dependences of TSZ width and the boundary of coagulation zone on the velocity of the fibre movement into the biotissue for the radiation power $5 \mathrm{~W}$.

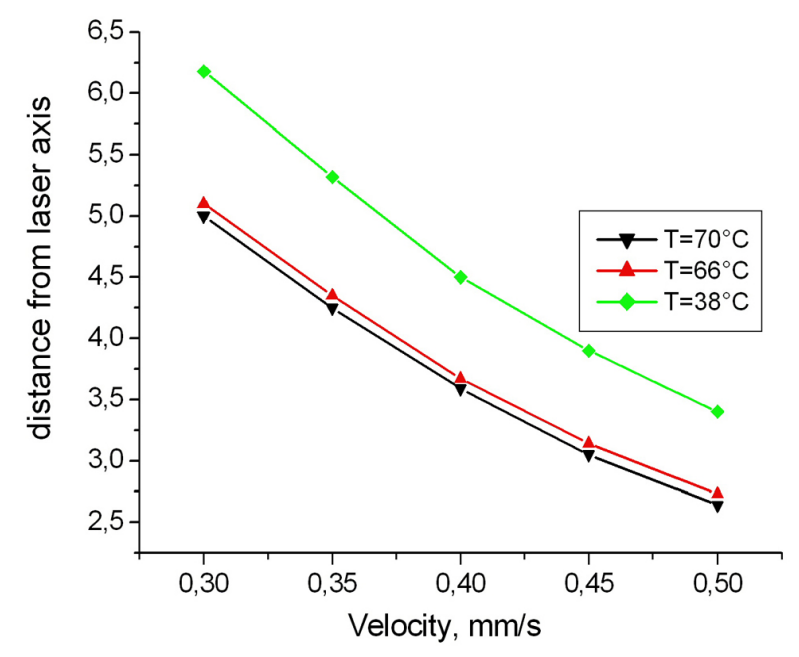

Fig. 7 Dependences of the TSZ width and the coagulation zone boundary position on the velocity of the optical fibre movement into the depth of biotissue at the power $5 \mathrm{~W}$.

As follows from Fig. 6, good correlation is observed between the experimental and theoretical dependence for the tissue coagulation zone boundary. At the constant perforation velocity the TSZ width increases with the laser power and its boundary shifts into the tissue from the axis of the laser channel. At the constant power of radiation, the TSZ width decreases with the increase of the perforation velocity (Fig. 7), and its boundary shifts towards the axis of the laser channel.

\section{Conclusions}

The possibility to control the position and parameters of the zones of biotissue thermal stimulation depending on the conditions of the laser perforation in application to the problem of laser-induced regeneration of liver is investigated. For the fixed radiation power, with the increase of the fibre motion velocity the zones of thermal stimulation approach the axis of the laser channel. For the fixed velocity of the fibre motion, the same result is provided by the reduction of the laser radiation power, which offers the possibility of controlling the position of thermal stimulation zones.

\section{Disclosures}

All authors declare that there is no conflict of interests in this paper.

\section{Acknowledgment}

The work was carried out under support from the Federal Agency for Scientific Research (agreement No. 007-ГЗ/Ч3363/26) in the part of developing the experimental techniques and from the Russian Foundation for Basic Research (project No. 15-2904816) in the rest part. 